\title{
ENERGÍA SOLAR Y MARGINACIÓN. ANÁLISIS DE LA PERCEPCIÓN SOCIAL SOBRE NUEVAS TECNOLOGÍAS PARA LA ARTICULACIÓN DE UNA TRANSICIÓN ENERGÉTICA EN EL MUNICIPIO DE NEZAHUALCÓYOTL, MÉXICO
}

\author{
Ángel Raúl ARENAS AQUINO ${ }^{1 *}$, Yasuhiro MATSUMOTO KUWABARA² y Mina KLEICHE-DRAY3
}

${ }^{1}$ Departamento de Metodología de la Ciencia, Centro de Investigación y de Estudios Avanzados. Avenida Instituto Politécnico Nacional 2508, San Pedro Zacatenco, Ciudad de México, México, C. P. 07360

${ }^{2}$ Departamento de Ingeniería Eléctrica, Centro de Investigación y de Estudios Avanzados. Avenida Instituto Politécnico Nacional 2508, San Pedro Zacatenco, Ciudad de México, México, C. P. 07360

${ }^{3}$ Institut de Recherche pour le Développement-Ceped \& IFRIS. Rue Jacob 19, París, Francia, C. P. 75006

*Autor para correspondencia: fisangelr@gmail.com

(Recibido abril 2016; aceptado octubre 2016)

Palabras clave: tecnología fotovoltaica, desarrollo social, percepción ciudadana

\section{RESUMEN}

Nezahualcóyotl es el segundo municipio más poblado del Estado de México. La marginación es una cuestión social de gran influencia para el desarrollo urbano. Se planteó un estudio para analizar la intervención de la percepción social en la factibilidad de una transición energética basada en tecnología fotovoltaica para el municipio, por medio de técnicas socioestadísticas. La hipótesis supone diferentes tendencias de la población (dependiendo del nivel de marginación), para aceptar nuevas tecnologías energéticas e impulsar el bienestar de su comunidad. Se evaluaron las interacciones sociales y se analizó la estructuración de la percepción ambiental y económica sobre la energía solar. Se encontró que el nivel de marginación, los costos de la energía eléctrica y el conocimiento de nuevas tecnologías son factores que influyen la transición energética en el municipio. Existe desinformación sobre las consecuencias de una transición energética. La representación local de nuevas tecnologías es frágil y las prioridades sociales están vinculadas estrechamente con las comodidades domésticas.

Key words: photovoltaic technology, social development, public perception

\begin{abstract}
Nezahualcoyotl is the second largest municipality of the State of Mexico. Marginalization is a social issue of great influence on urban development. A study through socialstatistical techniques was conducted in order to analyze social perception intervention in energy transition feasibility for the municipality, based on photovoltaic technology. The hypothesis scenario assumes different tendencies for people to accept new energy technologies to promote community's welfare, depending on the marginalization level. Inhabitants' social interactions were evaluated and the environmental and organization of economic perception regarding solar energy was analyzed. It was found that marginalization level, electric energy costs and new technologies knowledge are factors that influence the municipality energy transition. There is misinformation about
\end{abstract}


the consequences of energy transition. The local representation of new technologies is fragile and social priorities are closely linked to household comforts.

\section{INTRODUCCIÓN}

La zona Metropolitana del Valle de México (ZMVM) es el área urbana más grande y poblada del país. Está delimitada por 16 delegaciones de la Ciudad de México con 8851080 hab y 59 municipios del Estado de México con 11168301 hab (INEGICONAPO 2012). En 2010, México emitió $750 \mathrm{MMt}$ de $\mathrm{CO}_{2}$ equivalente (SEMARNAT-INE 2012). Las fuentes ubicadas dentro de la ZMVM representaron el $7.7 \%$ de las emisiones nacionales de gases efecto invernadero (GEI). En ese mismo año la ZMVM demandó una energía total equivalente a 706 PJ (SEDEMA 2012), siendo 4756.8 PJ la demanda nacional total. Se manejan las cifras de 2010 debido a que son los números más recientes del inventario de emisiones de la ZMVM durante la realización de este trabajo.

El municipio de Nezahualcóyotl en el Estado de México es el segundo más poblado de dicha entidad (CONAPO 2012a). Las cifras de emisiones de GEI del municipio son escasas y poco claras, pero según la Secretaría del Medio Ambiente de la Ciudad de México (SEDEMA), se estima que en 2010 el municipio emitió entre 200000 y $500000 \mathrm{t}$ de $\mathrm{CO}_{2}$ equivalente. Esto lo convierte uno de los tres municipios con mayores emisiones en el estado. Por otro lado, el consumo de energía eléctrica per cápita para el año 2010 fue de $0.5 \mathrm{MWh}$, mientras que el consumo eléctrico del sector residencial fue de 335 $569 \mathrm{MWh}$ (INEGI 2010). No obstante, a pesar de que el crecimiento económico y desarrollo social se han visto favorecidos en la última década, el nivel de marginación continúa siendo un problema primordial.

El Consejo Nacional de Población (CONAPO 2012a) describe la marginación como "el conjunto de desventajas sociales acumulables de una localidad que configuran escenarios cada vez más desfavorables donde no están presentes ciertas oportunidades para el desarrollo y existen privaciones a bienes y servicios fundamentales para el bienestar". Dadas las actuales condiciones ambientales y la crisis social latente, es necesario que se recurra a fuentes alternas de energía y se fomenten políticas que permitan su uso masivo. Así, los efectos y procesos del deterioro ambiental asociados con el crecimiento urbano y con el metabolismo negativo de la propia ciudad podrían reducirse (Lazcano-Martínez 2005).
Se requiere un tipo particular de tecnología que permita su uso sin la dependencia directa de la red convencional de electricidad, que pueda satisfacer las necesidades de una edificación, que pueda acoplarse a las dimensiones urbanas compactas, que utilice una fuente de energía renovable y limpia y que dicha fuente esté disponible con la menor intermitencia posible. Es decir, que se pueda extraer energía de ella sin tener tantas interrupciones naturales o técnicas. Así, se puede pensar en la tecnología eólica y la tecnología solar. Sin embargo, la intermitencia del viento en zonas urbanas, así como el crecimiento de la importancia económica de la tecnología fotovoltaica (FV) gracias a la constante disminución de sus precios (PV Insights 2014), hace que la fuente solar responda a los requerimientos.

México se encuentra en su totalidad territorial dentro del llamado "cinturón solar" que es una de las zonas con mayor incidencia solar en el planeta, con radiación superior a $5 \mathrm{kWh} / \mathrm{m}^{2} / \mathrm{d}$ (Alemán-Nava et al. 2014). Este gran potencial de energía renovable puede ser aprovechado por la tecnología solar FV. Los sistemas FV son competitivos económicamente para electrificar zonas vulnerables (Cassedy 2000). Dichas poblaciones generalmente no demandan tanta potencia, esto hace que la mayoría de las veces sea menos costoso instalar dispositivos FV que extender los tendidos tradicionales de la red eléctrica. El uso de energía solar FV reporta una serie de ventajas desde el punto de vista energético y económico. Por ejemplo, los paneles FV se pueden montar en los techos de las viviendas (si no los tiene, los paneles pueden desempeñarse como uno) o en sus patios (si los tiene) y son capaces de generar toda la electricidad en una vivienda, tiene una elevada calidad energética, es inagotable a escala humana, no requieren sofisticar las medidas de seguridad, su balance energético es positivo generalmente a partir del tercer al quinto año, son de mantenimiento sencillo, con variedad de dimensiones, además de que aumentan el valor de la propiedad. Asimismo, existen inconvenientes como la inversión inicial de instalación (que es alta), para aumentar la capacidad se requieren mayores espacios, es más costoso al necesitar más módulos y no se puede almacenar la energía generada de forma directa, siendo necesario realizar una transformación energética (SánchezPacheco 2009). 
Aunque las repercusiones tecnoambientales afectan a todos los sectores socioeconómicos, los más afectados son y serán las poblaciones marginadas debido a su alta dependencia de los recursos que los rodean y su limitada posibilidad de adaptación tanto a las condiciones energéticas actuales como a las futuras (Juárez-Neri 2003).

Este trabajo plantea un estudio de caso para evaluar la factibilidad de una eventual transición energética urbana basada en tecnología FV para el sector habitacional del municipio de Nezahualcóyotl. Dicha evaluación realizada a través de un estudio socioestadístico en donde la opinión de los habitantes del municipio consolidará los resultados del análisis (Vélez 2001). La hipótesis del análisis supone diferentes tendencias de la población a aceptar nuevas tecnologías energéticas para impulsar el bienestar propio y de su comunidad, dependiendo del nivel de marginación. Los objetivos son evaluar las tendencias socio-estadísticas de los habitantes del municipio dependiendo del nivel de marginación, analizar la articulación de la percepción ambiental y económica sobre la tecnología FV y revelar las interacciones locales entre los intereses tecnoambientales, los de vivienda y sobre la transición energética.

Así, la importancia de esta investigación radica en la preocupación del desarrollo social de las regiones marginadas, la mitigación del impacto ambiental y el interés por comprender la percepción de dichos habitantes sobre sus necesidades.

\section{MARCO TEÓRICO ESTUDIO DE CASO: NEZAHUALCÓYOTL}

Un área geoestadística básica (AGEB) urbana es un conjunto de manzanas perfectamente delimitadas por calle y el uso del suelo es habitacional, industrial, de servicios o comercial. En las AGEB es posible relacionar la información estadística con el espacio geográfico y gracias a ellas se facilitan las actividades de captación de información (INEGI 2013).

De acuerdo con el documento oficial de integración municipal (Presidencia de Nezahualcóyotl 2013), Nezahualcóyotl comprende para su organización territorial y de gobierno: la cabecera municipal, la unidad administrativa y 111 colonias. Hay varias razones por las que se eligió el municipio de Nezahualcóyotl como estudio de caso:

\section{a. Marginación}

Este indicador se define como la dificultad de propagar el progreso técnico, así como la exclusión del proceso de desarrollo y sus beneficios en una comunidad. Nezahualcóyotl ha registrado un grado más bajo de marginación comparado con el indicador estatal, con un índice de -1.75 contra -0.6 respectivamente (GEDOMEX 2012). Las AGEB urbanas en promedio indican un nivel de marginación medio (Cuadro I). b. Índice de desarrollo humano (IDH)

El IDH mide las oportunidades de las personas para que gocen de una vida larga y saludable, accedan a conocimientos individuales y socialmente útiles, y con ello obtengan los medios suficientes para decidir sobre su entorno. Durante el periodo de 2000 a 2010, en Nezahualcóyotl, este indicador ascendió de 0.8177 a 0.8621 , esta cifra rebasó al indicador estatal (GEDOMEX 2012).

\section{c. Pobreza}

La pobreza se entiende como las carencias que tienen personas o comunidades, de las cuales depende su desarrollo y bienestar social. En el año 2010, alrededor de 6.5 MMhab del Estado de México vivían en situación de pobreza. De éstos, $7 \%$ se asentaban en Nezahualcóyotl (CONEVAL 2012). El municipio en la actualidad ha superado parte de esa problemática social. Se registró que $38.7 \%$ de la población total vive en algún tipo pobreza (GEDOMEX 2012).

CUADRO I. NIVEL DE MARGINACIÓN DEL MUNICIPIO DE NEZAHUALCÓYOTL (CONAPO 2012B, INEGI 2010)

\begin{tabular}{|c|c|c|c|c|c|c|}
\hline \multirow{2}{*}{ Municipio } & \multirow{2}{*}{ AGEB urbana } & \multicolumn{5}{|c|}{ Grado de marginación urbana } \\
\hline & & Muy alto & Alto & Medio & Bajo & Muy bajo \\
\hline \multirow{4}{*}{ Nezahualcóyotl } & 177 & --- & 18 & 138 & 3 & 18 \\
\hline & Dol & \multicolumn{5}{|c|}{ Grado de marginación urbana } \\
\hline & 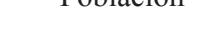 & Muy alto & Alto & Medio & Bajo & Muy bajo \\
\hline & 1104579 & --- & 102695 & 913950 & 17031 & 70903 \\
\hline
\end{tabular}

AGEB = área geoestadística básica 


\section{d. Condiciones de las viviendas}

La Comisión Nacional de Vivienda (CONAVI) considera una vivienda "carente" si presenta al menos una de las siguientes características: piso de tierra, techo de lámina o cartón y/o muros de materiales no duraderos. En 2010, en Nezahualcóyotl hubo en promedio 3.9 hab/vivienda (Cuadro II; GEDOMEX 2012). Para 2010 las viviendas con electricidad tuvieron el $98 \%$ de cobertura (GEDOMEX 2012).

CUADRO II. TIPO DE VIVIENDAS Y OCUPANTES EN ESTADO DE MÉXICO Y NEZAHUALCÓYOTL 2000-2010 (INEGI 2010)

\begin{tabular}{lcccc}
\hline Ámbito & Año & $\begin{array}{c}\text { Total de } \\
\text { viviendas }\end{array}$ & $\begin{array}{c}\text { Ocupantes de } \\
\text { las viviendas }\end{array}$ & $\begin{array}{c}\text { Hab/ } \\
\text { vivienda }\end{array}$ \\
\hline $\begin{array}{l}\text { Estado } \\
\text { de México }\end{array}$ & 2000 & 2743144 & 12472648 & 4.55 \\
& 2010 & 3100599 & 14953514 & 4.06 \\
\hline Nezahualcóyotl & 2000 & 274984 & 1195913 & 4.4 \\
& 2010 & 280513 & 1093307 & 3.9 \\
\hline
\end{tabular}

Existen trabajos de la trasformación urbano-rural en la zona centro de la ZMVM (Escamilla y Santos 2012) y sobre la segregación residencial en la ZMVM del año 2000 al 2010 (Pantaleón 2012). En cuestiones ambientales Torres-Carral (2011) discute la territorialidad y sustentabilidad urbana en la ZMVM y la insuficiencia de la misma. Por otro lado, se hace evidente que no existe una planificación integral relacionada con la gestión ambiental en el Valle de México (Lezama 2012). Sin embargo, a la fecha, no existe ningún estudio o investigación que relacione el nivel de marginación, la percepción tecnosocial y la transición energética basada en tecnología FV tanto para la ZMVM como para el municipio.

Los paradigmas de investigación sobre la percepción social tienen diversos enfoques, pero resulta importante la percepción de los problemas ambientales. La teoría crítica destaca la posición de los sujetos en la estructura social, la importancia de las relaciones sociales en la configuración de las preocupaciones ambientales y la necesidad de integrar políticas ambientales con políticas sociales y económicas (Catalán-Vázquez y Jarillo-Soto 2010).

\section{MATERIALES Y MÉTODOS}

\section{Determinación de la muestra}

Para determinar el tamaño de la muestra, la vivienda se desempeñó como entidad compleja para ser el elemento muestral, pero el elemento para definir el tamaño de la muestra fue la cantidad de individuos que habitan en la vivienda. De esta manera, las 27 preguntas que se realizaron abarcaron las características sociotécnicas de la vivienda, así como la toma de decisiones dentro de la misma (Cuadro III). Por lo tanto, el grupo preferencial a encuestar fueron personas mayores de edad con la facultad de tomar decisiones económicas para la vivienda.

\section{CUADRO III. Preguntas para la encuesta}

\section{¿En qué colonia vive?}

¿Cuántas personas habitan su vivienda?

¿Tiene electricidad en su casa?

¿Cómo califica el servicio de electricidad del gobierno?

¿Cuántos focos tiene en su casa?

¿De qué tipo son?

¿Cómo califica la iluminación natural de su casa?

Mencione si en su casa tiene:

8 Refrigerador

9 Microondas

10 Lavadora

11 Licuadora

12 Estufa de gas o eléctrica

13 Radio

14 TV

15 Computadora

16 TV de paga

17 Internet

18 Si se va la luz en su casa ¿cuántas veces se va al mes?

19 ¿Considera que estas fallas eléctricas afectan su calidad de vida?

20 En promedio, ¿al bimestre paga menos de \$ 200 de electricidad?

21 ¿Qué tanto le interesa el medio ambiente?

22 ¿Ha escuchado hablar de la energía solar o los paneles FV?

23 ¿Le interesaría comprar estos aparatos por 14000 pesos y prácticamente dejar de pagar luz?

24 Estos aparatos ocuparían una superficie de dos por dos metros ¿tendría espacio en su techo para instalarlos?

25 Se pueden conectar estos aparatos al cableado del gobierno y vender la electricidad sobrante que produzca, ¿preferiría venderla a sus vecinos o que el gobierno se la reembolse a su factura?

26 ¿Podría comprar con su dinero los aparatos o preferiría recibir financiamiento externo?

27 ¿Quién preferiría que le diera el financiamiento: banco, gobierno o asociación civil?

Para determinar el tamaño de la muestra se utilizó la ecuación para poblaciones finitas conocidas (Spiegel y Stephens 2005):

$$
\mathrm{n}=\frac{\mathrm{N} \cdot \mathrm{Z}_{\alpha}^{2} \cdot \mathrm{p} \cdot \mathrm{q}}{\mathrm{d}^{2}(\mathrm{~N}-1)+\mathrm{Z}_{\alpha}^{2} \cdot \mathrm{p} \cdot \mathrm{q}}
$$


Donde:

$\mathrm{N}=$ total de la población $=1104579$ hab

$\mathrm{Z} \alpha=$ nivel de confianza $=1.96$ (para $\alpha=0.05$, nivel de confianza de $95 \%$ )

$\mathrm{p}=$ proporción esperada $=0.5$ (proporción de $50 \%$ )

$\mathrm{q}=1-\mathrm{p}=1-0.5=0.5$

$\mathrm{d}=$ error $=0.02($ error de $2 \%)$

El nivel de confianza $Z \alpha$ se basó en que es una distribución normal relativamente simétrica. Por lo tanto, el tamaño de la muestra fue $\mathrm{n}=2396$.

\section{Muestreo}

Para que los habitantes de los cuatro niveles de marginación estuvieran representados en la encuesta, se garantizó que a su vez todas las colonias y AGEB estuvieran presentes en la muestra. Debido a la cantidad limitada de tiempo y recursos (un sólo encuestador), no se pudo recurrir a la visita casa por casa. Por lo tanto, para maximizar la cantidad de respuestas en el menor tiempo posible y garantizando la representatividad, se eligieron once puntos geográficos en los que se levantaron las encuestas con técnicas de muestreo no probabilístico por cuotas (los estratos sociales se identificaron por la mayoría de edad y con la facultad de tomar decisiones económicas para la vivienda), intencional (los grupos focales son las familias que acuden al punto establecido que cuentan con un miembro mayor de edad y con la facultad de tomar decisiones económicas para la vivienda), causal (los elementos seleccionados se eligieron en el punto mientras pasaban frente al encuestador) y con muestreo probabilístico estratificado, aleatorio simple y por conglomerados. Esto hace que el muestreo general sea mixto y por etapas múltiples (Spiegel y Stephens 2005, Hernández-Sampieri et al. 2006).

El criterio para elegir los once puntos geográficos yace en que estas ubicaciones son zonas de encuentro social que generalmente las familias del municipio visitan. Así, se pudo abarcar de manera aleatoria a la población de las cuatro zonas de marginación y se garantizó una heterogeneidad de las personas entrevistadas. Para encontrar los once puntos se utilizó el mapa digital de México del Instituto Nacional de Estadística y Geografía (INEGI 2014).

\section{Encuesta}

Las encuestas se realizaron del 1 de junio al 31 de julio de 2015 en diferentes días de la semana, dentro de un horario que oscilaba entre las $10 \mathrm{y}$ las $21 \mathrm{~h}$. Al finalizar una jornada de entrevistas se capturó la información en la base de datos. Lo que permitió discernir sobre el progreso y la cantidad de información faltante.

En el momento que se alcanzó el objetivo muestral cercano a 2396 (2392), se advirtió que aún faltaban colonias por muestrear, así como representatividad de las zonas de marginación de nivel alto. En ese momento se suspendieron las encuestas con el primer método y se pasó a una segunda etapa de muestreo.

En la segunda etapa se identificaron las colonias, AGEB y zonas de marginación faltantes. Para esta fase se requirió que el encuestador visitara esos sectores e hiciera entrevistas casa por casa. En esta etapa se levantaron las encuestas con técnicas de muestreo probabilístico estratificado, aleatorio simple y por conglomerados, además de muestreo no probabilístico por cuotas e intencional. Se obtuvieron 338 muestras adicionales para tener un total de 2730. Las preguntas se orientaron a la razón tecnológica de los equipos FV. Fue necesario considerar que los encuestados podrían desconocer todos los datos que se les solicitaban, por lo que se formularon preguntas que evitarán vacíos significativos en la información. Para ello se explicó y describió de manera general la información pertinente para que tuvieran un panorama en donde apoyar sus respuestas. No se pretendió que la encuesta fuera un instrumento para obtener respuestas favorables hacia la transición energética o la tecnología FV ni mucho menos de persuadir a los residentes de Nezahualcóyotl. Las variables con las que se trabajaron son tanto cuantitativas como cualitativas. Todas las respuestas arrojaron variables discretas.

Las preguntas se dividieron en cinco grupos:

1. Grupo 1 , preguntas 1 y 2 : La pregunta 1 es la referencia para ubicar el AGEB de acuerdo con la colonia y así conocer el nivel de marginación. La pregunta 2 sirvió para cotejar la cantidad de habitantes con la información oficial de acuerdo con el AGEB.

2. Grupo 2, preguntas 3 a 7: La pregunta 3 es para cotejar la información de la AGEB sobre acceso a la electricidad. Las preguntas 4 a 7 sirvieron para evaluar la percepción del servicio y el conocimiento de las características de su vivienda.

3. Grupo 3, preguntas 8 a 17: Preguntas testigo para comparar con los resultados oficiales y establecer un posible desfase de resultados.

4. Grupo 4, preguntas 18 a 20: Percepción de calidad de vida en función de la calidad del servicio. La pregunta 22 considera el consumo promedio de una vivienda del municipio con 4 hab (Fthenakis et al. 2008, GEDOMEX 2012, Raugei et al. 2012) 
5. Grupo 5, preguntas 21 a 27: Conocimiento técnico y ambiental de la tecnología solar FV. Se evaluó el interés y apropiación de una nueva tecnología. La pregunta 23 basó la información en la consideración general de una tasa de retorno de 11.5 años para un sistema FV considerando que se paga el servicio eléctrico a MXN\$ 200 bimestrales (Fthenakis et al. 2008, Raugei et al. 2012).

Las 2730 muestras se obtuvieron con un total de 553 encuestas, éstas se dividen por AGEB (Cuadro IV).

El despliegue de resultados se estructuró en dos bloques independientes a los grupos de preguntas, de acuerdo con su naturaleza numérica y con el enfoque metodológico:

\section{CUADRO IV. ÁREA GEOESTADÍSTICA BÁSICA (AGEB) POR NIVEL DE MARGINACIÓN (INEGI 2010)}

\begin{tabular}{lcccc}
\hline Nivel marginación & Alto & Medio & Bajo & Muy bajo \\
\hline Ageb & 23 & 464 & 24 & 42 \\
Porcentaje & 4.16 & 83.91 & 4.34 & 7.59 \\
\hline
\end{tabular}

- El primer bloque contiene las preguntas con respuestas de calificación del uno al cinco, así como las preguntas que requieren un análisis estadístico reflexivo derivado del comportamiento de los datos.

- El segundo bloque contiene las preguntas con respuestas si/no que sirven como testigo para contrastar con la información oficial, y que no precisan de una inferencia estadística.
Para el primer bloque se obtuvieron las medidas de tendencia central para realizar la interpretación estadística (Spiegel y Stephens 2005; Cuadro V, Cuadro VI).

Los niveles de marginación se clasificaron con un numeral: alto $=1$, medio $=2$, bajo $=3$ y muy bajo $=4$.

Para realizar la interpretación y análisis estadístico de los datos cualitativos, se utilizó el programa de estadística R-CRAN. Las comparaciones se basaron en las variables testigo para cotejar con la información obtenida oficialmente por las instituciones gubernamentales. Se requiere entender el comportamiento de los datos obtenidos en función del nivel de marginación.

Se utilizó la prueba de Kruskal-Wallis (Field et al. 2012) para comprobar si existen diferencias significativas entre las medias de las muestras de la encuesta. Además, se realizó una regresión lineal múltiple y se obtuvo el coeficiente de determinación $\mathrm{R}^{2}$ (Field et al. 2012) para determinar la relación entre las variables analizadas y el interés para adquirir un sistema FV.

\section{RESULTADOS Y DISCUSIÓN}

\section{Primer bloque de preguntas}

Se obtuvo que el promedio del nivel de marginación en el municipio fue 2.15 , que se considera nivel medio de marginación (Cuadros $\mathbf{V}$ y VI), esto coincidió con la información estadística oficial. Para la pregunta 4 , la calificación de la calidad del servicio de electricidad del gobierno, la media fue 2.43, que indica insatisfacción en la población encuestada.

CUADRO V. Medidas de tendencia central, preguntas 1, 2, 4 y 5

\begin{tabular}{|c|c|c|c|c|}
\hline $\begin{array}{l}\text { Medida } \\
\text { tendencia central }\end{array}$ & $\begin{array}{c}\text { Pregunta } 1 \\
\text { Marginación }\end{array}$ & $\begin{array}{c}\text { Pregunta } 2 \\
\text { Hab/vivienda }\end{array}$ & $\begin{array}{c}\text { Pregunta } 4 \\
\text { Calidad servicio }\end{array}$ & $\begin{array}{c}\text { Pregunta } 5 \\
\text { cantidad focos }\end{array}$ \\
\hline Media & 2.15 & 4.94 & 2.43 & 7.53 \\
\hline $\begin{array}{l}\text { Desviación } \\
\text { estándar }\end{array}$ & 0.60 & 1.70 & 1.12 & 3.85 \\
\hline Frecuencia 1 & 23 & \multirow{5}{*}{-} & 140 & \multirow{5}{*}{ - } \\
\hline Frecuencia 2 & 464 & & 158 & \\
\hline Frecuencia 3 & 24 & & 150 & \\
\hline Frecuencia 4 & 42 & & 88 & \\
\hline Frecuencia 5 & - & & 17 & \\
\hline $\begin{array}{l}\text { No sabe / no } \\
\text { contestó }\end{array}$ & - & - & - & 11 \\
\hline
\end{tabular}


CUADRO VI. MEDIDAS DE TENDENCIA CENTRAL, PREGUNTAS 7, 18 Y 21

\begin{tabular}{lccc}
\hline $\begin{array}{l}\text { Medida } \\
\text { tendencia } \\
\text { central }\end{array}$ & $\begin{array}{c}\text { Pregunta 7 } \\
\text { Calidad } \\
\text { iluminación }\end{array}$ & $\begin{array}{c}\text { Pregunta 18 } \\
\text { Frecuencia } \\
\text { de falla }\end{array}$ & $\begin{array}{c}\text { Pregunta 21 } \\
\text { Interés por } \\
\text { el ambiente }\end{array}$ \\
\hline $\begin{array}{l}\text { Media } \\
\text { Desviación }\end{array}$ & 2.78 & 2.09 & 2.56 \\
estándar & 1.29 & 0.96 & 1.17 \\
Frecuencia 1 & 114 & 185 & 133 \\
Frecuencia 2 & 135 & 171 & 127 \\
Frecuencia 3 & 115 & 160 & 137 \\
Frecuencia 4 & 127 & 25 & 127 \\
Frecuencia 5 & 58 & 6 & 15 \\
No sabe / & & 5 & 14 \\
no contestó & 4 & & \\
\hline
\end{tabular}

La media de focos por vivienda fue 7.43, esto quiere decir que las casas encuestadas gozan de servicio eléctrico adecuado para poseer esa cantidad de focos, aunque también es un indicador del nivel de marginación, del cual se aprecia un nivel medio y alto de acceso a los servicios de iluminación.

La población calificó de "regular" la calidad de iluminación natural de sus viviendas con una media de 2.78. Esto se relaciona con una aparente necesidad de mayor número de focos (pregunta 5). La media de la frecuencia mensual de fallas eléctricas fue 2.09, por lo que se entiende que la calificación del servicio del gobierno no haya sido alta. La media de la pregunta 21 (2.56), indica que la población encuestada tiene poco interés por el ambiente.

Se verificó la diferencia entre las muestras del primer bloque por nivel de marginación. Para ello se realizó una prueba de Kruskal-Wallis entre cada grupo de variables, tomando las muestras como los niveles de marginación (Cuadro VII). Entonces, es probable que las medias de los grupos de la encuesta por nivel de marginación sean estadísticamente indistinguibles y provengan de la misma población.

\section{Segundo bloque de preguntas}

Todas las viviendas encuestadas tienen servicio eléctrico, sin embargo, no todas están conectadas a la red legalmente y otras que sí tienen conexión legal aún no tienen contrato con la Comisión Federal de Electricidad (CFE).

Se pone en evidencia de la pregunta 12 , que la población no tiene como prioridad el uso de la electricidad como medio de preparación de alimentos, al igual que la posibilidad de que el poder adquisitivo no permita adquirir otra clase de estufa debido al costo más elevado. Por otro lado, todas las viviendas de la muestra tienen televisión y radio. La gran mayoría tiene licuadora, lavadora, microondas y refrigerador. Y poco más de la mitad tiene acceso a computadora, televisión de paga e internet (Fig. 1). Estos son indicadores del nivel de marginación. No obstante, estos datos indican que la población muestreada considera que sus necesidades de entretenimiento así como de comodidad son importantes, incluso por encima de la capacidad adquisitiva. Hay viviendas que tienen televisión, pero no tienen refrigerador (Cuadro VIII). Los resultados de la variable relacionada con la cantidad de focos en la vivienda corresponden con la información oficial obtenida por INEGI (2015; Cuadro IX).

La sección referente a la tecnología FV se ilustra en las preguntas 19, 20, 22, 23 y 24. En primer lugar, poco más de la mitad de la muestra considera que su calidad de vida sí se ve afectada por las fallas eléctricas. Sumado a eso, la gran mayoría paga más de MXN\$ 200 al bimestre. Sin embargo, más de la mitad no conoce ni ha oído hablar sobre la energía solar o los paneles FV. Cabe resaltar que 353 viviendas cuentan con espacio disponible para instalar un sistema $\mathrm{FV}$, pero al parecer más de la mitad no invertiría MXN\$ 14000 en él.

A pesar de que la población no muestra un gran interés por el ambiente y la mayoría no invertiría en un sistema $\mathrm{FV}$, prefieren vender directamente a otros usuarios la electricidad residual que generaría el sistema FV en lugar de inyectarla a la red pública y recibir un descuento en el recibo.

Se encontró que más de la mitad de la muestra no sabe si requeriría un financiamiento, esto da más solidez a la idea del poco interés en el ambiente y en la tecnología solar. A pesar de ello, 170 viviendas sí solicitarían financiamiento. De éstas 170, la mitad preferiría una asociación civil como proveedora de financiamiento, por encima de un banco o del gobierno.

En este bloque también se realizó una prueba de Kruskal-Wallis, pero en este caso se cotejaron las variables de las muestras obtenidas de la encuesta con las variables testigo de las estadísticas oficiales. Sin embargo, no se obtuvieron diferencias significativas (Cuadro X).

Finalmente, se realizó una regresión lineal múltiple en R-CRAN para identificar el efecto que tienen los grupos de respuestas sobre el interés de adquirir un sistema FV. La variable dependiente para la regresión fue el grupo de datos de la variable 24 (interés en comprar sistema FV) y las variables independientes fueron los datos de todos los demás grupos de variables (en esta regresión se ignoran los datos de las variables 8 a 18 porque fueron utilizadas como variables testigo). 
CUADRO VII. RESULTADOS DE PRUEBA KRUSKAL-WALLIS PARA PRIMER BLOQUE

\begin{tabular}{|c|c|c|c|c|c|c|c|}
\hline \multirow{2}{*}{$\frac{\text { Pregunta }}{4}$} & \multicolumn{2}{|c|}{ Niveles de marginación } & \multirow{2}{*}{$\begin{array}{c}\text { Valor } \mathrm{p} \\
0.557\end{array}$} & \multirow{2}{*}{$\frac{\text { Pregunta }}{23}$} & \multicolumn{2}{|c|}{ Niveles de marginación } & \multirow{2}{*}{$\begin{array}{l}\text { Valor } \mathrm{p} \\
0.424\end{array}$} \\
\hline & 1 & 2 & & & 1 & 2 & \\
\hline & 1 & 3 & 0.502 & & 1 & 3 & 0.111 \\
\hline & 1 & 4 & 0.792 & & 1 & 4 & 0.335 \\
\hline & 2 & 3 & 0.312 & & 2 & 3 & 0.325 \\
\hline & 2 & 4 & 0.174 & & 2 & 4 & 0.300 \\
\hline & 3 & 4 & 0.140 & & 3 & 4 & 0.548 \\
\hline \multirow[t]{6}{*}{7} & 1 & 2 & 0.932 & \multirow[t]{6}{*}{24} & 1 & 2 & 0.232 \\
\hline & 1 & 3 & 0.366 & & 1 & 3 & 0.787 \\
\hline & 1 & 4 & 0.179 & & 1 & 4 & 0.291 \\
\hline & 2 & 3 & 0.912 & & 2 & 3 & 0.912 \\
\hline & 2 & 4 & 0.050 & & 2 & 4 & 0.364 \\
\hline & 3 & 4 & 0.396 & & 3 & 4 & 0.727 \\
\hline \multirow[t]{6}{*}{19} & 1 & 2 & 0.297 & \multirow[t]{6}{*}{25} & 1 & 2 & 0.750 \\
\hline & 1 & 3 & 0.692 & & 1 & 3 & 0.870 \\
\hline & 1 & 4 & 0.669 & & 1 & 4 & 0.699 \\
\hline & 2 & 3 & 0.172 & & 2 & 3 & 0.180 \\
\hline & 2 & 4 & 0.585 & & 2 & 4 & 0.236 \\
\hline & 3 & 4 & 0.369 & & 3 & 4 & 0.599 \\
\hline \multirow[t]{6}{*}{20} & 1 & 2 & 0.632 & \multirow[t]{6}{*}{26} & 1 & 2 & 0.553 \\
\hline & 1 & 3 & 0.870 & & 1 & 3 & 0.285 \\
\hline & 1 & 4 & 0.699 & & 1 & 4 & 0.129 \\
\hline & 2 & 3 & 0.170 & & 2 & 3 & 0.291 \\
\hline & 2 & 4 & 0.246 & & 2 & 4 & 0.109 \\
\hline & 3 & 4 & 0.579 & & 3 & 4 & 0.579 \\
\hline \multirow[t]{6}{*}{21} & 1 & 2 & 0.136 & \multirow[t]{6}{*}{27} & 1 & 2 & 0.752 \\
\hline & 1 & 3 & 0.917 & & 1 & 3 & 0.414 \\
\hline & 1 & 4 & 0.098 & & 1 & 4 & 0.369 \\
\hline & 2 & 3 & 0.740 & & 2 & 3 & 0.287 \\
\hline & 2 & 4 & 0.179 & & 2 & 4 & 0.802 \\
\hline & 3 & 4 & 0.447 & & 3 & 4 & 0.778 \\
\hline \multirow{6}{*}{22} & 1 & 2 & 0.882 & & & & \\
\hline & 1 & 3 & 0.213 & & & & \\
\hline & 1 & 4 & 0.933 & & & & \\
\hline & 2 & 3 & 0.175 & & & & \\
\hline & 2 & 4 & 0.653 & & & & \\
\hline & 3 & 4 & 0.327 & & & & \\
\hline
\end{tabular}

Los resultados muestran que el modelo explica $67 \%$ de la variación de la variable dependiente. Además, existe una relación significativa entre las variables del modelo de regresión ( $\mathrm{p}<0.05$; Cuadro XI).

Por lo tanto, del cuadro XI, se nota que cinco variables tienen un efecto significativo en el interés o posibilidad de adquirir un sistema FV. Las variables con mayor representatividad en orden de significancia (de mayor a menor), son:

1. Interés en solicitar financiamiento para adquirir sistema FV (variable 27).

2. Nivel de marginación (variable 1).
3. Pago de electricidad menor a MXN\$200 (variable 21).

4. Conocimiento previo de tecnología FV (variable 23).

5. Interés en el ambiente (variable 22).

Los resultados de la regresión indican que el nivel de marginación, los costos actuales de la energía eléctrica y el conocimiento de nuevas tecnologías son factores de alta importancia para la transición energética en el municipio. Es menos probable que la población con nivel alto y medio de marginación (que es el mayor porcentaje de habitantes en Nezahualcóyotl) 
Pregunta 6: Tipos de foco

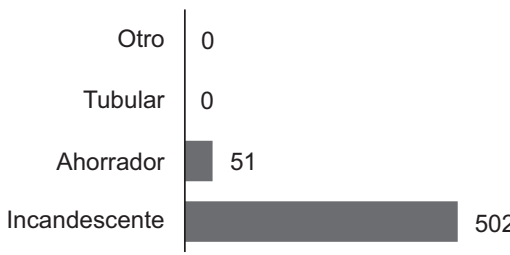

Preguntas 8 a 17

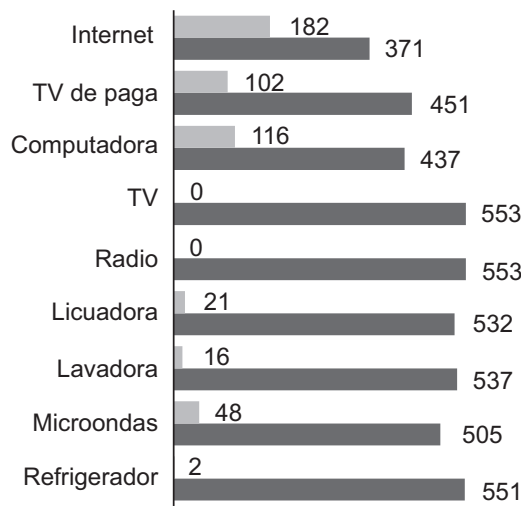

$\square$ No $\square \mathrm{Si}$

Preguntas 25: Vender o reembolso

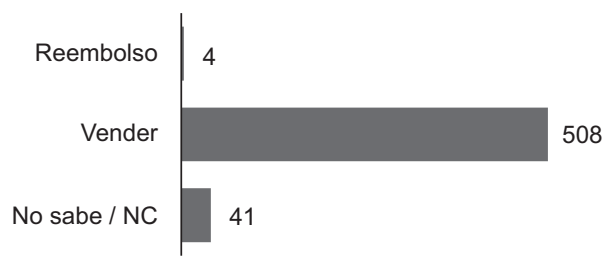

Pregunta 12: Tipos de estufa

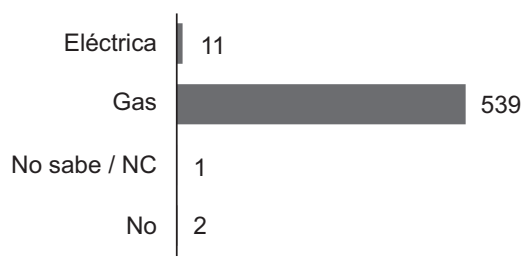

Preguntas 19, 20, 22, 23 y 24

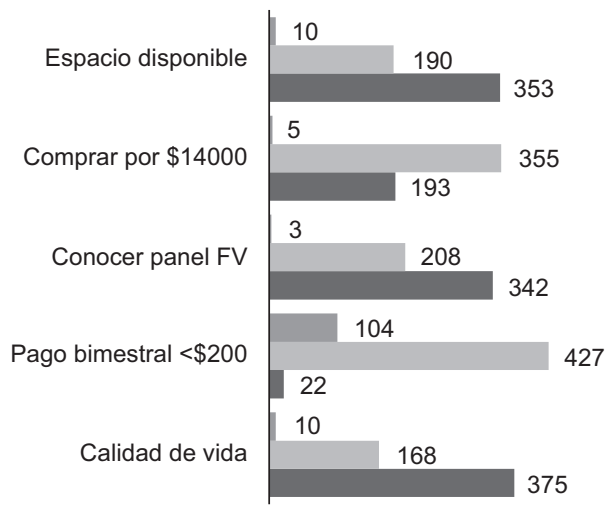

$\square$ No sabe / NC $\square$ No $\square \mathrm{Si}$

Preguntas 26: Tipo de financiamiento

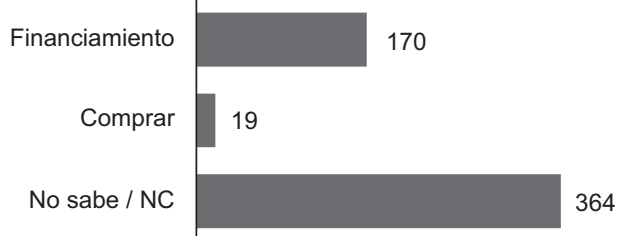

Preguntas 27: Procedencia de financiamiento

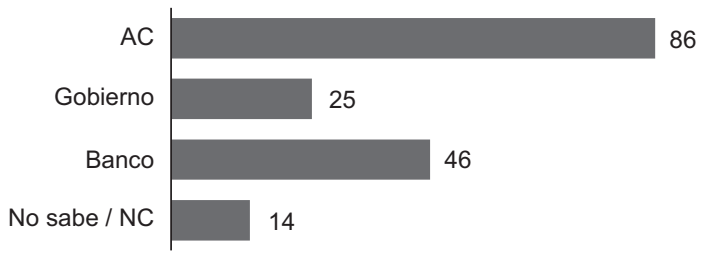

Fig. 1. Histogramas de frecuencia del segundo bloque de preguntas

considere promover un cambio de paradigma energético. Sin embargo, los incentivos económicos incrementan las posibilidades. Por el contrario, los habitantes con bajos niveles de marginación tienen más probabilidades de aceptar nuevas tecnologías que impliquen una transición energética. Estos tres factores principales en conjunto son los que se tienen que considerar primordialmente para fomentar la transformación tecnosocial.

Es claro que la población del municipio no está en condiciones de pobreza energética. Además, los hogares que se encuentran en situación de marginación 
CUADRO VIII. DATOS DE POSESIÓN DE APARATOS, INSTITUTO NACIONAL DE ESTADÍSTICA Y GEOGRAFÍA (INEGI 2015) vs. ENCUESTA

\begin{tabular}{|c|c|c|c|c|c|}
\hline \multirow[t]{2}{*}{ Variable } & \multirow[t]{2}{*}{ Tipo de bien } & \multicolumn{2}{|c|}{ INEGI (\%) } & \multicolumn{2}{|c|}{ Encuesta (\%) } \\
\hline & & SI & NO & SI & NO \\
\hline 8 & Refrigerador & 90.03 & 9.52 & 99.64 & 0.36 \\
\hline 9 & Horno de microondas & 53.08 & 46.47 & 54.25 & 45.75 \\
\hline 10 & Lavadora & 77.93 & 21.61 & 79.93 & 20.07 \\
\hline 13 & Radio & 85.68 & 13.72 & 88.79 & 11.21 \\
\hline 14 & Televisión & 97.95 & 1.48 & 100 & - \\
\hline 15 & Computadora & 40.42 & 59.03 & 45.21 & 54.79 \\
\hline 16 & Televisión de paga & 25.12 & 74.22 & 29.66 & 70.34 \\
\hline 17 & Internet & 44.25 & 55.18 & 50.45 & 49.55 \\
\hline 18 & Teléfono celular & 78.71 & 20.74 & 82.28 & 17.72 \\
\hline
\end{tabular}

CUADRO IX. NÚMERO DE FOCOS EN LA VIVIENDA, DATOS DE INSTITUTO NACIONAL DE ESTADÍSTICA Y GEOGRAFÍA(INEGI 2015) vs. ENCUESTA

\begin{tabular}{lcccc}
\hline \multicolumn{5}{c}{ Número de focos en la vivienda } \\
\hline $\begin{array}{l}6-5 \\
\text { focos }\end{array}$ & $\begin{array}{c}11-15 \\
\text { focos }\end{array}$ & focos & $\begin{array}{c}16-20 \\
\text { focos }\end{array}$ & $\begin{array}{c}21 \text { focos } \\
\text { y más }\end{array}$ \\
\hline \multicolumn{5}{c}{ INEGI } \\
\hline 153707 & 98420 & 19034 & 5324 & 2301 \\
$55.00 \%$ & $35.22 \%$ & $6.81 \%$ & $1.90 \%$ & $0.82 \%$ \\
\hline $45.57 \%$ & $39.06 \%$ & $10.13 \%$ & $1.99 \%$ & $1.27 \%$ \\
\hline
\end{tabular}

media tienden a desarrollar prácticas más armónicas al sortear con creatividad las restricciones económicas.

La televisión es el aparato más importante, por lo que el entretenimiento es una prioridad cultural.

La aparente disposición de las personas a utilizar mecanismos de ahorro económico sugiere que este comportamiento es de gran utilidad para tratar aspectos como la transición energética y la apropiación tecnológica.

La inacción de los habitantes sobre temas de elección se debe a que retrasan la intención de cambio porque prefieren cualquier opción que tienen actualmente y porque existe un costo inherente al cambio.

Los encuestados no creen que el interés en el ambiente sea algo común, sigue viéndose como una actividad acotada. Aquí entra otro factor importante a considerar además de la comodidad, la economía.

De acuerdo con la apreciación de la mayoría de los encuestados, para que la información que reciban sea útil, debe ser contextualizada y adaptada a sus circunstancias personales. La mejor manera para
CUADRO X. RESULTADOS DE PRUEBA KRUSKALWALLIS PARA SEGUNDO BLOQUE

\begin{tabular}{|c|c|c|c|}
\hline Pregunta & \multicolumn{2}{|c|}{ Niveles de marginación } & \multirow{2}{*}{$\frac{\text { Valor } p}{0.3259}$} \\
\hline \multirow{6}{*}{2} & 1 & 2 & \\
\hline & 1 & 3 & 0.3405 \\
\hline & 1 & 4 & 0.5882 \\
\hline & 2 & 3 & 0.3293 \\
\hline & 2 & 4 & 0.3068 \\
\hline & 3 & 4 & 0.8386 \\
\hline \multirow{6}{*}{5} & 1 & 2 & 0.7778 \\
\hline & 1 & 3 & 0.6163 \\
\hline & 1 & 4 & 0.2923 \\
\hline & 2 & 3 & 0.1507 \\
\hline & 2 & 4 & 0.4976 \\
\hline & 3 & 4 & 0.3583 \\
\hline \multirow{6}{*}{6} & 1 & 2 & 0.4582 \\
\hline & 1 & 3 & 0.2901 \\
\hline & 1 & 4 & 0.1144 \\
\hline & 2 & 3 & 0.353 \\
\hline & 2 & 4 & 0.486 \\
\hline & 3 & 4 & 0.367 \\
\hline
\end{tabular}

describir la eficiencia energética y las nuevas tecnologías es haciéndolo en términos de ahorro monetario.

\section{CONCLUSIONES}

Los puntos trascendentales encontrados son la desinformación ciudadana relacionada con transición energética, la representación local del uso de nuevas tecnologías, las prioridades sociales vinculadas con las comodidades domésticas y la percepción urbana de la administración gubernamental.

Los resultados indican que en el municipio de Nezahualcóyotl hay diferentes tendencias de 
CUADRO XI. RESULTADOS DE REGRESIÓN LINEAL MÚLTIPLE PARA TODAS LAS VARIABLES

\begin{tabular}{lrcccc}
\hline Variable & Estimado & Error estándar & Valor t & Probabilidad $>|t|$ & REP \\
\hline Pregunta 1 & -0.081 & 0.028 & -2.901 & 0.004 & $* *$ \\
Pregunta 2 & -0.011 & 0.007 & -1.449 & 0.148 & \\
Pregunta 4 & -0.005 & 0.012 & -0.419 & 0.675 & \\
Pregunta 5 & 0.005 & 0.004 & 1.452 & 0.147 & \\
Pregunta 6 & -0.079 & 0.043 & -1.809 & 0.071 & \\
Pregunta 7 & -0.005 & 0.009 & -0.495 & 0.621 & \\
Pregunta 19 & 0.010 & 0.013 & 0.727 & 0.467 & $*$ \\
Pregunta 20 & -0.017 & 0.025 & -0.688 & 0.491 & $*$ \\
Pregunta 21 & -0.045 & 0.016 & -2.807 & 0.005 & $*$ \\
Pregunta 22 & -0.021 & 0.010 & -2.065 & 0.039 & $*$ \\
Pregunta 23 & 0.057 & 0.026 & 2.185 & 0.029 & $*$ \\
Pregunta 25 & 0.003 & 0.025 & 0.140 & 0.888 & $*$ \\
Pregunta 26 & 0.026 & 0.044 & 0.589 & 0.556 & $* 2 \mathrm{e}-16$ \\
Pregunta 27 & -0.422 & 0.014 & -30.833 & & \\
& Valor R del modelo: 0.6781 & & \\
\hline
\end{tabular}

La columna "REP = representatividad" marca con asteriscos las variables con mayor representatividad. Entre más asteriscos, mayor representatividad

aceptación de nuevas tecnologías en función del nivel de marginación.

La población del municipio considera que el servicio eléctrico no es bueno, pero es suficiente para cumplir con los estándares familiares para tener una calidad de vida donde las necesidades básicas puedan satisfacerse y la comodidad sea alcanzable. Las familias que tienen un enfoque de ahorro energético no ponen en práctica esta perspectiva de la forma más efectiva. En estos hogares predominantemente existe la opinión de que se ubican en un nivel bajo de marginación. No obstante, la conciencia ambiental y energética no implica el interés en la transición energética.

Cuando existe un beneficio económico, las personas adquieren una apreciación diferente sobre tecnología desconocida o fuera de su interés. No se puede hablar de una resistencia a lo "nuevo".

Queda claro que en Nezahualcóyotl las personas están acostumbradas a que su vida transcurra sin cambios significativamente positivos para su calidad de vida.

Además, se hace presente el desconocimiento de muchas problemáticas a las que las familias están expuestas, desde la cuestión de los subsidios eléctricos, el funcionamiento de la tecnología FV y el tema del financiamiento. Un problema esencial es que las familias estén conscientes de la precariedad en la que viven, así como de sus carencias sociales. Esto no tiene que ver con la clasificación gubernamental de los estratos sociales, tiene que ver con la apreciación de la aparente comodidad y la representación de las necesidades propias. Es indispensable entender la representación social de sus necesidades. Las prioridades que las comunidades asignen a los requerimientos cotidianos definirán el balance de las decisiones gubernamentales con las políticas públicas.

Por otro lado, hay carencia de información en temas de energía y ambiente. La población no tiene acceso a información relacionada con la tecnología FV o la energía solar o incluso con los sistemas energéticos convencionales. A pesar de que las familias parecen tener una preocupación genuina sobre el ambiente, hace falta profundidad en estos saberes.

Sin embargo, hay interés en aprender sobre temas que atraigan beneficios. El desconocimiento de muchos temas no parece mermar el interés de la población en adquirir nueva información. La población aprecia el interés más profundo en sus necesidades y desea que el gobierno tenga ese mismo interés.

Los procesos de información gobierno-sociedad son de una sola vía, es decir, sólo uno de los participantes envía la información y el otro la recibe, sin que haya retroalimentación. Además, la información provista es generalizada y por lo tanto, las familias la perciben como irrelevante. Entonces, los habitantes toman decisiones complejas basadas en información incompleta, sin tener el lujo de poder calcular las implicaciones.

Es muy importante ser consciente del verdadero origen de las dificultades socioeconómicas de las familias y evitar responsabilizar injustamente, de lo contrario, la racionalización técnica puede llevar a limitar el margen de maniobra en los hogares. 
Localmente una transición energética asistida por tecnología FV representa una independencia energética que debería dotar de mayor libertad a los ciudadanos, además significaría restar una carga al gobierno federal.

Un elemento emergente como estudio futuro para el municipio es el de los derechos ambientales, incluyendo el de apropiación de la naturaleza. En ese contexto, los derechos ambientales se definen como derechos a autogobernarse conforme a sus cosmovisiones, usos y costumbres. Lo que implica el derecho de cada pueblo a establecer sus propias prácticas de uso y transformación de sus recursos naturales (Leff 2001).

La barrera más grande para la aceptación y propagación de la tecnología solar son los mitos e ideas falsas que existen en las mentes de los potenciales adoptadores, siendo las más comunes que las energías renovables son caras y que no son capaces de satisfacer la demanda energética.

La solución a la problemática debe ser discutida desde la perspectiva de las mayorías, pero también considerando la visión de quienes gobiernan. Los esfuerzos contra la degradación ambiental desde una perspectiva ecosocial es una condición sin la que no es posible la sustentabilidad urbana, esto detonaría la revolución energética y el aprovechamiento de nuevas tecnologías.

\section{REFERENCIAS}

Alemán-Nava G., Casiano-Flores V., Cárdenas-Chávez D., Díaz-Chávez R., Scarlat N., Mahlknecht J., Dallemand J.F. y Parra R. (2014). Renewable energy research progress in Mexico: A review. Renew. Sust. Energ. Rev. 32, 140-153. DOI: 10.1016/j.rser.2014.01.004

Cassedy E.S. (2000). Prospects for sustainable energy: A critical assessment. Cambridge University Press. Cambridge, Reino Unido, 284 pp.

Catalán-Vázquez M. y Jarillo-Soto E. (2010). Paradigmas de investigación aplicados al estudio de la percepción pública de la contaminación del aire. Rev. Int. Contam. Ambie. 26 (2), 165-178.

CONEVAL (2012). Informe de evaluación de la política de desarrollo social en México 2012. Consejo Nacional de Evaluación de la Política de Desarrollo Social [en línea]. http://www.coneval.org.mx/Evaluacion/ Paginas/IEPDS_2014.aspx 15/02/2014.

CONAPO (2012a). Índice de marginación por localidad 2010, colección: Índices sociodemográficos. Consejo Nacional de Población [en línea]. http://www.conapo. gob.mx/ES/CONAPO/Indice_de_Marginacion_por_ Localidad_2010 15/02/2014.
CONAPO (2012b). Índice de marginación urbana 2010, colección: Índices sociodemográficos. Consejo Nacional de Población [en línea]. http://www.conapo. gob.mx/en/CONAPO/Indice_de_marginacion_urbana 2010 15/02/2014.

Escamilla I. y Santos C. (2012). La zona metropolitana del Valle de México: transformación urbano-rural en la región centro de México. Memorias. XII Coloquio internacional de geocrítica. Bogotá, Colombia, 7 al 12 de mayo, 2012, 1-15.

Field A., Miles J. y Field Z. (2012). Discovering statistics using R. SAGE publications, Londres, Inglaterra, 992 pp. DOI: 10.1111/insr.12011_21

Fthenakis V.M., Kim H.C. y Alsema E. (2008). Emissions from photovoltaic life cycles. Environ. Sci. Technol. 42 (6), 2168-2174. DOI: 10.1021/es071763q

GEDOMEX (2012). Programa de desarrollo regional macro región III oriente región IX Nezahualcóyotl 2006-2011. Gobierno del Estado de México [en línea]. http://docplayer.es/20476608-Programa-de-desarrolloregional-macro-region-iii-oriente-region-ix-nezahualcoyotl.html 10/02/2015.

Hernández-Sampieri R., Fernández-Collado C. y Baptista P. (2006). Metodología de la Investigación. 4a ed. McGrawHill. México D.F., México, 850 pp.

INEGI (2010). Censo Nacional de Vivienda 2010. Instituto Nacional de Estadística y Geografía [en línea]. http://www3.inegi.org.mx/sistemas/ageburbana 28/01/2014.

INEGI-CONAPO (2012). Delimitación de las zonas metropolitanas de México 2010. Instituto Nacional de Estadística y Geografía - Consejo Nacional de Población [en línea]. http://www.conapo.gob.mx/es/ CONAPO/Zonas_metropolitanas_2010 5/03/2014.

INEGI (2013). Marco geoestadístico nacional. Instituto Nacional de Estadística y Geografía [en línea]. http://www3.inegi.org.mx/sistemas/biblioteca/ficha. aspx?upc=702825292829 17/02/2014.

INEGI (2014). Mapa digital de México. Instituto Nacional de Estadística y Geografía [en línea]. http://gaia.inegi. org.mx/mdm6 28/01/2014.

INEGI (2015). Panorama sociodemográfico, encuesta intercensal 2015. Instituto Nacional de Estadística y Geografía [en línea] http://www3.inegi.org.mx/sistemas/tabuladosbasicos/default.aspx? $\mathrm{c}=33725 \& \mathrm{~s}=\mathrm{est}$ 08/02/2016

Juárez-Neri V.M. (2003). Condiciones de la vivienda en la zona metropolitana del Valle de México en el año 2000 [en línea]. http://www.ub.edu/geocrit/sn/sn-146(040). htm 28/01/2018.

Lazcano-Martínez M. (2005). El acceso al suelo y a la vivienda de los sectores informales: el caso de la ciudad de México. Revista INVI. 20 (54), 18-54. 
Leff E. (2001). Justicia ambiental. Construcción y defensa de los nuevos derechos ambientales, culturales y colectivos en América Latina. Programa de las Naciones Unidas para el Medio Ambiente - Centro de Investigaciones Interdisciplinarias en Ciencias y Humanidades de la Universidad Nacional Autónoma de México. México D.F., México, 275 pp.

Lezama J. (2012). Gobernar la metrópoli: El caso de la gestión ambiental en el Valle de México. [en línea]. http://www.iea.usp.br/pesquisa/programas-e-projetosatuais/estudos-comparados-sao-paulo-e-mexico/ GobernarlaMetrpoliLagestinambientalenlaZMVM. pdf 24/11/2014.

Pantaleón N. (2012). Segregación residencial en la zona metropolitana del Valle de México, 2000-2010, un análisis espacial. Tesis de Maestría. Facultad Latinoamericana de Ciencia Sociales (FLACSO). México D.F., México, 126 pp.

Presidencia de Nezahualcóyotl (2013). Presentación del bando municipal de Nezahualcóyotl [en línea]. http://neza.gob.mx/docs/bandompal_2013_v1.pdf 7/04/2015.

PV Insights (2014). Precios de contado de módulos y celdas solares [en línea]. http://pvinsights.com 2/6/2014.

Raugei M., Fullana-I-Palmer P. y Fthenakis V. (2012). The energy return on energy investment (EROI) of photovoltaics: Methodology and comparisons with fossil fuel life cycles. Energ. Policy. 45, 576-582. DOI: 10.1016/j.enpol.2012.03.008
Sánchez-Pacheco C. (2009). Sistemas de energía solar fotovoltaica aplicados a viviendas residenciales en entorno urbano. Tesis de Maestría. Universidad Internacional de Andalucía. Andalucía, España, 63 pp.

SEMARNAT-INE (2012). Inventario nacional de emisiones de gases de efecto invernadero 1990-2010. Secretaría de Medio Ambiente y de Recursos Naturales - Instituto Nacional de Ecología [en línea]. http://www. inecc.gob.mx/descargas/cclimatico/inf_inegei_public_2010.pdf 24/03/2014.

SEDEMA (2012). Inventario de Emisiones de la Zona Metropolitana del Valle de México. Secretaría de Medio Ambiente de la Ciudad de México [en línea]. https:// www.gob.mx/cms/uploads/attachment/file/110143/ Inventario-emisioneszmvm2012.pdf 8/02/2014.

Spiegel M.R. y Stephens J. (2009). Estadística. 4a ed. Mc Graw-Hill. México D.F., México, 577 pp.

Torres-Carral G. (2011). Territorialidad y sustentabilidad urbana en la Zona Metropolitana del Valle de México. Econ. Soc. Territ. 11 (36), 317-347.

DOI: $10.22136 /$ est002011108

Vélez C.M. (2001). Apuntes de metodología de la investigación, un resumen de las principales ideas para el desarrollo de proyectos de investigación. Universidad Escuela de Administración, Finanzas e Instituto Tecnológico. Medellín, Colombia, 59 pp. 